\title{
A NOTE ON THE LITTLEWOOD-TAUBER THEOREM
}

\section{L. GLASSER ${ }^{1}$}

The application of Wiener's generalized Tauberian Theorem [1] to a sequence of functions in $L_{1}(-\infty, \infty)$ leads to the following.

Lemma. Let $K_{0}$ belong to $L_{1}(-\infty, \infty)$ and be such that

$$
\int_{-\infty}^{\infty} K_{0}(x) e^{i u x} d x \neq 0, \quad-\infty<u<\infty .
$$

Let $g$ be bounded on $(-\infty, \infty)$ and let

$$
\lim _{x \rightarrow \infty} \int_{-\infty}^{\infty} K_{0}(x-y) g(y) d y=A \int_{-\infty}^{\infty} K_{0}(y) d y .
$$

Then if $\left\{K_{n}(x)\right\}$ is a sequence of functions in $L_{1}$ such that for $n \rightarrow \infty$

$$
\int_{-\infty}^{\infty} K_{n}(x-y) g(y) d y \rightarrow I(x)
$$

for almost all $x$ and

we have

$$
\int_{-\infty}^{\infty} K_{n}(y) d y \rightarrow I
$$

$$
\lim _{x \rightarrow \infty} I(x)=A I
$$

As an application of this lemma we give a particularly simple proof of the Littlewood-Tauber Theorem [2].

Theorem. Let $\sum_{0}^{\infty} a_{n} x^{n}$ converge to $f(x)$ for $|x|<1$ and let

$$
\lim _{x \rightarrow 1-} f(x)=s<\infty .
$$

If $n\left|a_{n}\right|<K<\infty$, then $\sum_{0}^{\infty} a_{n}=s$.

Proof.

$$
\begin{aligned}
\left|\sum_{0}^{N} a_{n}-\sum_{0}^{\infty} a_{n} e^{-n / N}\right| & \leqq \sum_{0}^{N}\left|a_{n}\right|\left(1-e^{-n / N}\right)+\sum_{N+1}^{\infty}\left|a_{n}\right| e^{-n / N} \\
& \leqq K\left(2+\sum_{N+1}^{\infty} \frac{e^{-n / N}}{n}\right)<\infty \quad \text { for } N>1,
\end{aligned}
$$

Received by the editors August 15, 1967 and, in revised form, September 27, 1967.

1 The author is grateful to Professor J. Karamata for a communication and Dr. S. Zimering for his comments. 
hence $s(u)=\sum_{0}^{[u]} a_{n}$ is bounded. Now,

$$
\begin{aligned}
f\left(e^{-x}\right) & =x \int_{0}^{\infty} e^{-u x} s(u) d u \\
& =\int_{0}^{\infty} e^{-(\xi-y)} e^{-e^{-(\xi-y)}} s\left(e^{y}\right) d y,
\end{aligned}
$$

where $x=e^{-\xi}$. Thus,

$$
s \equiv \lim _{x \rightarrow 0^{+}} f\left(e^{-x}\right)=\lim _{\xi \rightarrow \infty} \int_{-\infty}^{\infty} K_{0}(\xi-y) s\left(e^{y}\right) d y,
$$

where $K_{0}(x)=e^{-x} e^{-e^{-x}}$. We have

$$
\int_{-\infty}^{\infty} K_{0}(x) e^{i u x} d x=\Gamma(1-i u) \neq 0
$$

Let $K_{n}(x)=(n / \pi)^{1 / 2} e^{-n x^{2}}$, then the conditions of the Lemma are satisfied under the hypothesis that $n\left|a_{n}\right|$ is bounded and, therefore, noting that in this case $I(x)=g(x), I=1$, we have

$$
s=\lim _{x \rightarrow \infty} \lim _{n \rightarrow \infty} \int_{-\infty}^{\infty} K_{n}(x-y) s\left(e^{y}\right) d y=\lim _{x \rightarrow \infty} s\left(e^{x}\right)=\sum_{0}^{\infty} a_{n} .
$$

\section{REFERENCES}

1. N. Wiener, The Fourier integral, Dover, New York, 1933; p. 73.

2. G. H. Hardy, Divergent series, Oxford Univ. Press, New York, 1949; p. 154.

Batelle Memorial Institute 